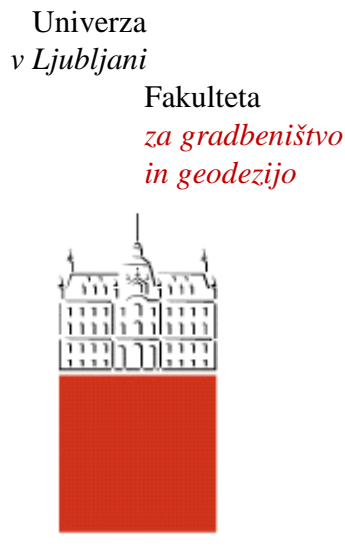

Jamova 2

1000 Ljubljana, Slovenija http://www3.fgg.uni-lj.si/

DRUGG - Digitalni repozitorij UL FGG http://drugg.fgg.uni-lj.si/

Ta članek je avtorjeva zadnja recenzirana različica, kot je bila sprejeta po opravljeni recenziji.

Prosimo, da se pri navajanju sklicujete na bibliografske podatke, kot je navedeno:
University

of Ljubljana

Faculty of

Civil and Geodetic

Engineering

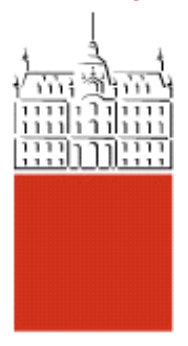

Jamova 2

SI - 1000 Ljubljana, Slovenia

http://www3.fgg.uni-lj.si/en/

DRUGG - The Digital Repository http://drugg.fgg.uni-lj.si/

This version of the article is author's manuscript as accepted for publishing after the review process.

When citing, please refer to the publisher's bibliographic information as follows: 


\title{
GPS-DERIVED GEOID USING ARTIFICIAL NEURAL NETWORK AND LEAST SQUARES COLLOCATION
}

\author{
B. Stopar, T. Ambrožič, M. Kuhar, G. Turk \\ University of Ljubljana, Faculty of Civil and Geodetic Engineering
}

\begin{abstract}
The geoid heights are needed for determining the orthometric heights from the Global Positioning System (GPS) ellipsoidal heights. There are several methods for geoid height determination. The paper presents a method employing the Artificial Neural Network (ANN) approximation together with the Least Squares Collocation (LSC). The surface obtained by the ANN approximation is used as a trend surface in the least squares collocation. In numerical examples four surfaces were compared: the global geopotential model (EGM96), the European gravimetric quasigeoid 1997 (EGG97), the surface approximated with minimum curvature splines in tension algorithm and the ANN surface approximation. The effectiveness of the ANN surface approximation depends on the number of control points. If the number of well distributed control points is sufficiently large, the results are better than those obtained by the minimum curvature algorithm and comparable to those obtained by the EGG97 model.
\end{abstract}

\section{INTRODUCTION}

The task of height determination presents one of the most compelling research areas related to GPS. The computation of orthometric heights with GPS (the so called GPSlevelling) is only possible with the knowledge of the ellipsoidal (GPS) heights and the geoid heights.

Nowadays, the gravimetric method is most commonly used technique for precise determination of the geoid. The necessary condition for its use is existence of high resolution gravity data sets. With the lack of gravity data the geoid determination is possible by means of various geometric methods, the astrogeodetic method and the method of determining the geoid heights from GPS in combination with geometric levelling.

The well known equation relates these three quantities:

$$
H=h-N
$$

where $N$ is either the geoid height or the quasigeoid height (height anomaly) and $H$ the orthometric or the normal height; $h$ is the ellipsoidal height.

In general, the shape of the geoid is very complex and the task of approximating the geoid surface by a relatively simple mathematical expression is hardly easy. For the local geoid approximation various interpolation surfaces may be used, e.g., low-order polynomials or even more complex surfaces, such as trigonometric functions and various spline functions, respectively. Alternatively, ANN used as an approximator is introduced [1].

In this paper a method using ANN for approximation of the local geoid surface is employed. This approximation surface is further refined by using the LSC approach.

Various types of ANNs are used for solving a large variety of problems. So far ANN was implemented in various scientific earth-science disciplines, such as in oceanography (sea level forecast), cartography (pseudocolor photomap production), remote sensing (e.g., classification of remotely-sensed multi-spectral imagery), GIS, GPS-positioning in integration with other sensors, seismology, and geodesy for solving problems in coordinate transformation, and in predicting Earth orientation parameters. 
Some of the recent publications [8], [9], [18] deal with the use of ANN in geoid approximation.

\section{LEAST SQUARES COLLOCATION}

If a fairly large number of well distributed points with known ellipsoidal and orthometric heights is available for an area, then the so called GPS-derived geoid can be determined. The method involves the development of a local geoid surface model using a surface fitting procedure. The local geoid surface fitting model is accomplished by taking $N$ as a function of the position of each point in the area under consideration:

$$
N=N(\phi, \lambda)
$$

where variables $\phi$ and $\lambda$ represent geodetic coordinates of points with known geoid heights, i.e. control points.

The artificial neural network (ANN) is employed in order to approximate this functional relation (Equation 2) by a trend surface. Unmodelled fine structure of the local geoid surface is further estimated by the least squares collocation (LSC).

The LSC is represented by the following equations [14], [15]:

$$
\mathbf{v}=\mathbf{l}-\mathbf{B} \Delta, \mathbf{v}=\mathbf{s}+\mathbf{r}
$$

where $\mathbf{l}$ consists of the geoid height values at control points, $\Delta$ are the parameters of approximation, i.e. the ANN approximation surface, the design matrix $\mathbf{B}$ includes geodetic coordinates of control points, whereas $\mathbf{v}, \mathbf{s}$, and $\mathbf{r}$ are residuals, signal and random noise, respectively.

The geoid heights on the new points - where only the GPS-height is known - can be calculated by the ANN approximation surface. The unmodelled part of the geoid height (signal) in the new points representing the fine relief of the local geoid may be afterwards predicted by the means of the LSC:

$$
\mathbf{s}=\mathbf{C D}^{-1}(\mathbf{l}-\mathbf{B} \Delta)
$$

The matrix $\mathbf{C}$ contains covariances between the geoid heights on new and control points in the area. The matrix $\mathbf{D}$ is the covariance matrix of observations $\mathbf{l}$, i.e., geoid heights, and it contains covariances between geoid heights at all control points. The matrix $\mathbf{D}$ is sum of the covariance matrix of the signal in control points and the covariance matrix of the random measuring errors (noise) taken as diagonal and constant.

For the computation of covariances the well-known autocorrelation function known as the third-order Markov undulation model was used [11]:

$$
C_{N N}(r)=C_{0}\left(1+\frac{r}{D}+\frac{r^{2}}{3 D^{2}}\right) e^{-\frac{r}{D}}
$$

where $r$ is the distance between points under consideration. The free parameters in models are the variance of the geoid height $C_{0}$ and the characteristic distance $D$ of the covariance function. For the purpose of predicting the signal component of the geoid height, the correlation distance is of interest. The correlation distance $\xi$ is the value of the argument of which $C(r)$ has decreased to half of its value at $r=0$ [15]:

$$
C(\xi)=\frac{1}{2} C_{0}
$$

In order to determine the optimal solution empirical covariances were computed and the model (6) was fitted to them. 
The basic idea and the motivation for the early development of ANNs was the study of the structure and processes in human brain that is in several aspects similar to ANN. An ANN and the human brain both have units called neurons which are interconnected. Similarly to a human brain, ANN has to be taught or trained. There are two types of learning procedures: supervised learning, in which questions and answers are known and where the ANN learns the correct answers; and unsupervised learning where the answers are not known. ANN is a network of simple units operating locally. The units are connected by connections reducing or amplifying the signal from one unit to another. Each unit receives signals from other units, processes these signals and transmits the signals to further units.

There are several types of ANN geometry. A review of different ANNs is given in several papers, books and internet sites e.g., [13], [17]. Usually, if functional approximation is sought, the multi-layer feed-forward network is chosen. Since it is our aim to approximate the geoid heights, the multi-layer feed-forward network trained by the supervised learning was chosen in this research.

\section{MULTI-LAYER FEED-FORWARD NETWORK}

The geometry of a multi-layer feed-forward neural network is shown in Figure 1. Input units (neurons) are connected to the first layer of hidden units that are further connected to the units of the second hidden layer. The units of the last hidden layer are connected to the output units. The multi-layer feed-forward networks are usually employed as the approximators of the unknown functional relation. In fact, it was shown by [4] and [7], that any continuous function may be accurately approximated by the multi-layer feed-forward neural network.

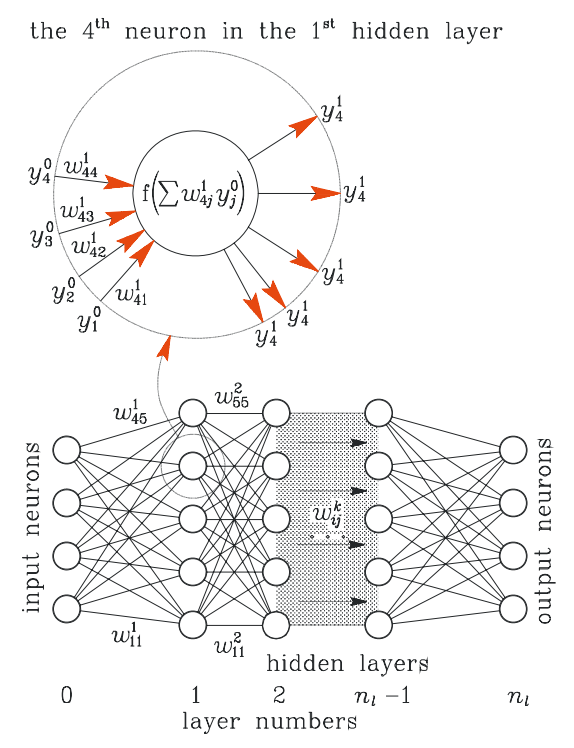

Fig. 1. The geometry of a multi-layer feed-forward neural network

The input units represent the input data, and the output units represent the output data. The hidden layers and all the connections between the units may be considered as a black box performing the necessary transformations of the input data so that the target output data are obtained. 
Each unit is represented by its value $y_{i}^{k}$, and each connection between the units is represented by its weight $w_{i j}{ }^{k}$. The index $i$ corresponds to the unit number of the $k^{\text {th }}$ layer, while index $j$ corresponds to the unit number of the $(k-1)^{\text {th }}$ layer. The input layer is denoted by 0 , and the output layer is denoted by $n_{l}$. The signals travel in one direction only, i.e., from the input layer toward the output layer. The value of a unit $y_{j}{ }^{k-1}$ is multiplied by the corresponding weight $w_{i j}{ }^{k}$ and added to the value of the signal in the unit of the next layer. In addition, the value of bias neuron or threshold $\vartheta_{i}{ }^{k}$ is added to the equation:

$$
y_{i}^{k}=f\left(\sum_{j=1}^{n_{k-1}} w_{i j}^{k} y_{j}^{k-1}+\vartheta_{i}^{k}\right)
$$

This equation is illustrated in Figure 1. Activation function $f($.) enables the modelling of an arbitrary non-linear relation between input and output variables. Different functions could be used as an activation function, the usual choices of the activation function are a sigmoid function:

$$
f(y)=\frac{1}{1+e^{-y}}
$$

tanh $y$, or Gaussian. The behaviour of the neural network depends on the values of the weights $w_{i j}{ }^{k}$ and thresholds $\vartheta_{i}{ }^{k}$ that have to be determined by the learning (training) procedure.

The set of known input and output values is termed an input-output pair. All inputoutput pairs are usually divided into three sets. The first is termed as learning or training set used to determine the connection weights $w_{i j}{ }^{k}$ and thresholds $\vartheta_{i}{ }^{k}$. When the learning procedure ends, i.e., when the neural network performs adequately for all input-output pairs in the learning set, the neural network is assessed on the validation set of input-output pairs and the optimal neural network is chosen. Finally, the chosen and taught neural network is tested, using the testing set of data.

In some cases the training procedure becomes ill-conditioned, i.e., if the input and/or output data are not normalized, see, e.g., [17]. Therefore, for numerical reasons the values of input and output units have to be normalized. The normalization of the values of output units depends on the range of activation function. Usually, the linear transformation works well, however if the data are clustered a non-linear transformation may help.

The supervised learning is in fact a general optimization problem in which the minimum of error $E_{p}$ is sought:

$$
E_{p}=\frac{1}{2} \sum_{i=1}^{n_{o}}\left(t_{p i}-y_{p i}^{n_{l}}\right)^{2}
$$

where $t_{p i}$ are the target output values, $y_{p i}^{n_{l}}$ are the values of neurons in the output layer $n_{l}$, i.e., the output values evaluated by $\mathrm{ANN}, n_{o}$ is the number of neurons in the output layer, i.e., the number of output variables.

This problem is numerically very demanding since there is usually a large number of local minima. There are two essentially different approaches: the error backpropagation algorithms that are basically a gradient method, and the genetic algorithm approach, which is in fact a stochastic search [5]. There are many variations and combinations of the above mentioned methods [20]. If the number of weights is relatively small, the gradient method is a favourable. The error back-propagation (or the generalized delta rule - as termed by [16] is a gradient method in which the weights are changed for a chosen step size in the direction of the maximum descent for each input-output pair. However, there is always the possibility of finding only a local minimum, which may be unable to provide a satisfactory set of weights. One solution 
for the problem is simply running the error back-propagation procedure from different starting points and then single out the best result.

In the error back-propagation approach the weights are changed in the direction of maximum descent of each input-output pair $p$

$$
\Delta w_{i j}^{k}=-\Delta_{w} \frac{\partial E_{p}}{\partial w_{i j}^{k}} \quad \text { and } \Delta \vartheta_{i}^{k}=-\Delta_{\vartheta} \frac{\partial E_{p}}{\partial \vartheta_{i}^{k}}
$$

where $\Delta_{w}$ is the step size defining the rate of changing the weights $w_{i j}{ }^{k}$, and $\Delta_{\vartheta}$ is the step size defining the rate of changing the thresholds $\vartheta_{i}{ }^{k}$. The derivatives in Equation (10) are determined consecutively from the weights between the output layer and the last hidden layer towards the weight between the input layer and the first hidden layer, i.e., by the chain rule

$$
\frac{\partial E_{p}}{\partial w_{i j}^{k}}=\frac{\partial E_{p}}{\partial y_{p i}^{k}} \frac{\partial y_{p i}^{k}}{\partial w_{i j}^{k}} \text { and } \frac{\partial E_{p}}{\partial \vartheta_{i}^{k}}=\frac{\partial E_{p}}{\partial y_{p i}^{k}} \frac{\partial y_{p i}^{k}}{\partial \vartheta_{i}^{k}}
$$

The derivatives $\partial y_{p i}{ }^{k} / \partial w_{i j}{ }^{k}$ and $\partial y_{p i}{ }^{k} / \partial \vartheta_{i}{ }^{k}$ are obtained from Equation (7)

$$
\frac{\partial y_{p i}^{k}}{\partial w_{i j}^{k}}=\frac{d f\left(y_{p i}^{\prime k}\right)}{d y_{p i}^{\prime k}} y_{p j}^{k-1} \quad \text { and } \quad \frac{\partial y_{p i}^{k}}{\partial \vartheta_{i}^{k}}=\frac{d f\left(y_{p i}^{\prime k}\right)}{d y_{p i}^{\prime k}}
$$

In the case of output layer neurons the derivatives $\partial E_{p} / \partial y_{p i}{ }^{k}$ are determined by the following equation stemming from (9)

$$
\frac{\partial E_{p}}{\partial y_{p i}^{n_{l}}}=-\left(t_{p i}-y_{p i}^{n_{l}}\right) \text {. }
$$

In the case of all other neurons the derivatives are obtained from Equation (7)

$$
\frac{\partial E_{p}}{\partial y_{p i}^{k-1}}=\sum_{j=1}^{n_{k}} \frac{\partial E_{p}}{\partial y_{p j}^{k}} \frac{\partial y_{p j}^{k}}{\partial_{p i}^{k-1}}
$$

The process is repeated for each input-output pair $p$ until the error is smaller than prescribed for all input-output pairs. If the prescribed error is too small, overfitting may occur. Overfitting means that the neural network may reproduce input-output pairs used in the learning procedure, but fails to generalize them and may as a consequence produce erroneous results, i.e. if some values of the input units are changed.

There are two major difficulties with using the error back-propagation: it is almost impossible to choose the optimal step size, and, additionally, quite often the procedure converges to a local minimum. If the step size is too large, we may overshoot the minimum. On the other hand, if the step size is too small, the convergence is very slow. Both difficulties may be overcome by different procedures with adaptive step size [10], or with the introduction of the inertial term [13].

The parameters, i.e., the number of hidden layers and the number of hidden neurons, of the optimal neural network are problem dependent. One of the methods for choosing the right network is using the validation set to determine which network performs best. However, some general guidelines can be given. If the number of units is large, the learning procedure may be slow, since each forward calculation needs a substantial computational effort. Although larger networks are usually able to learn the sought relationship, this may sometimes present a drawback. A large network may easily reproduce the training set of input-output pairs but fails to generalize, yielding to a poor testing performance. Networks with insufficient units may have problems with learning properly during the learning procedure. 


\section{APPLICATION OF MULTI-LAYER FEED-FORWARD NETWORK IN GEOID HEIGHT DETERMINATION}

In the case of geoid height determination the two input units represent the coordinates of the control point, whereas the only output unit represents the quasigeoid height of the control point. These three values constitute one input-output pair.

In the training procedure the weights $w_{i j}{ }^{k}$ and the treshold $\vartheta_{i}{ }^{k}$ are determined so that the geoid height determined by the ANN does not differ from the target value of the geoid height by more than a required value for all control points, i.e. for all inputoutput pairs. When the training procedure is completed the geoid height can be determined by the Equation (7) for any point inside the area under consideration.

Since there are two input units and only one output unit, the geometry of the neural network can be denoted by: $2 \times n_{1} \times n_{2} \times \cdots \times n_{n_{l-1}} \times 1$, denoting that there are 2 input units, $n_{1}$ units in the first hidden layer, $n_{2}$ units in the second hidden layer, . ., $n_{n_{l-1}}$ units in the last hidden layer and only a single unit in the output layer. Several geometries were tried and the optimal ones (for our specific application) are shown in Table 1.

\section{NUMERICAL EXAMPLES}

The method ANN-LSC was assessed for the data of the German Baden-Württemberg state area, where a GPS/levelling data set consisting of 125 points with known quasigeoid heights is available [21], (see Figure 2). In order to validate the proposed method, all results were compared to two different quasigeoid solutions: the global geopotential model EGM96 [12] and the European Gravimetric quasigeoid 1997 (EGG97) [3]. Because the ANN is of the type of a non-parametric interpolation method, it was also compared against one such interpolation technique, i.e. minimum (continuous) curvature splines in tension surface (MC), [19].

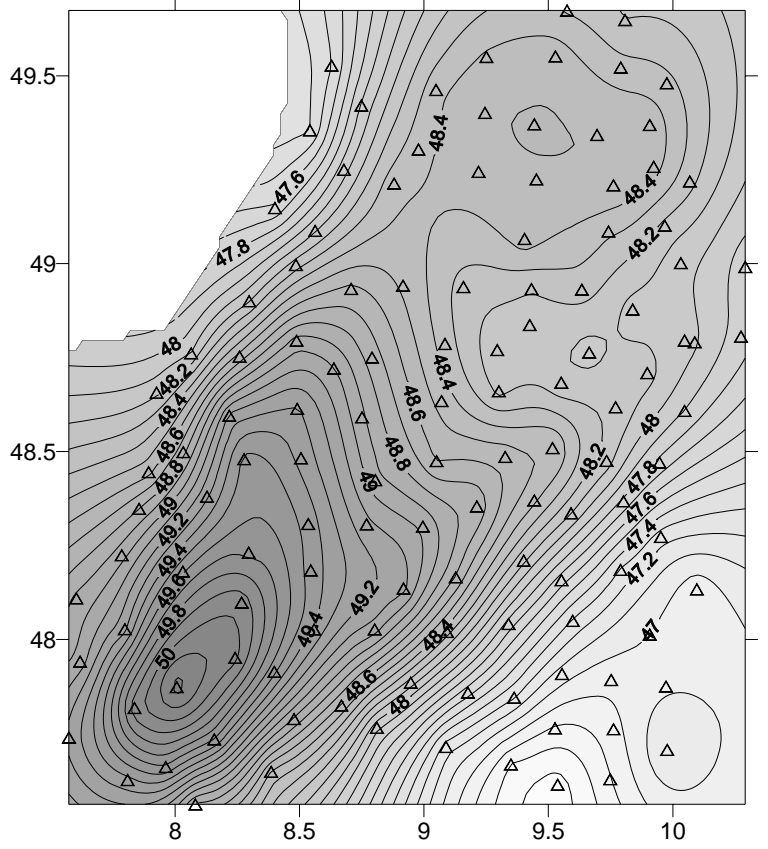

Fig. 2. Quasigeoid in Baden-Württemberg area

In the computation process the following two testing strategies were applied. In the first one quasigeoid heights were interpolated at the GPS/levelling points from the 
global EGM96 and continental EGG97 models, respectively. The "ANN surface" was determined using the described ANN algorithm. These three solutions were treated as a trend part of the quasigeoid surface. So it was possible to form residuals on height control points in the sense of:

$$
v_{i}=\zeta_{i}^{G P S}-\zeta_{i}^{M}=\left(h_{i}-H_{i}\right)-\zeta_{i}^{M}=s_{i}+r_{i}, \quad i=1, \ldots . n
$$

where $\zeta_{i}{ }^{M}$ is model quasigeoid undulation (ANN, EGM96 or EGG97), and $s_{i}$ and $r_{i}$ are signal and nosie, respectively (see Equation (3)).

In the case of EGM96 and EGG97 solutions, the trend component was further modelled by a 3-parameter datum shift $\Delta X, \Delta Y, \Delta Z$ determined from the residuals $v_{i}$ on the height control points. This model is described by the equation [2]:

$$
v_{i}=\cos \phi_{i} \cos \lambda_{i} \Delta X+\cos \phi_{i} \sin \lambda_{i} \Delta Y+\sin \phi_{i} \Delta Z, \quad i=1, \ldots n
$$

where $n$ is the number of control points.

Afterwards, the signal part of the GPS/leveling quasigeoid undulation $s$ was det ermined using the LSC algorithm.

On the contrary the minimum curvature interpolation method yields final solution which is then compared against other solutions. The interpolated surface generated by the minimum curvature is analogous to a thin, linearly plastic plate passing through each of the data values with a minimum amount of bending. For the computation we used the SURFER 8.0 software package [6]. The algorithm implements the concept of tension and also includes the regional trend approximation by removing a least-squares plane from the data [19]. The only parameter that can be varied in the data combination is the internal tension factor $T$. In all results, $\mathrm{MC}$-interpolated surface is generated with internal tension factor set to zero $(T=0)$, giving the minimum standard deviation of residuals in comparison to actual quasigeoid undulations $\zeta^{G P S}$ (see next section).

Four different numerical tests were performed with respect to the number of control (training) points used. In each test computation, the complete set of 125 points (inputoutput pairs) was divided into two subsets: the training set (input height control points) and the validation set. The basic data on the four test computation are summarized in Table 1.

Table 1. Training sets and optimal ANN geometry

\begin{tabular}{ccccc}
\hline Test & $\begin{array}{c}\text { No. of training } \\
\text { inp.-output pairs } \\
\text { (control points) }\end{array}$ & $\begin{array}{c}\text { No. of validation } \\
\text { inp.-output pairs } \\
\text { (new points) }\end{array}$ & $\begin{array}{c}\text { Optimal ANN } \\
\text { geometry }\end{array}$ & $\begin{array}{c}\text { No. of iterations } \\
\text { in ANN training }\end{array}$ \\
\hline 1 & 25 & 100 & $2 \times 30 \times 30 \times 1$ & 10857 \\
2 & 50 & 75 & $2 \times 25 \times 30 \times 1$ & 11806 \\
3 & 75 & 50 & $2 \times 25 \times 20 \times 1$ & 47352 \\
4 & 99 & 26 & $2 \times 20 \times 15 \times 1$ & 26677 \\
\hline
\end{tabular}

The error relative to the span of the target output values allowed in the ANN training process was set to $5 \%$, which is relatively large. This high value was chosen in order to avoid overfitting which could result in poor generalization.

Unfortunately, we could not include any reliable accuracy estimatations regarding the quantities involved in the computation. Documentation of the GPS/leveling data set in Baden-Württemberg provides no information about its accuracy. Since this is a local geoid analysis, levelling can be considered nearly error free, so the major part comes 
from the errors in GPS-heights. It was such decided to set the noise variance equal to $(1.5)^{2} \mathrm{~cm}^{2}$ which is nowadays easily achieved by GPS-measurements.

\section{RESULTS OF THE NUMERICAL TESTS}

Signal variance $C_{0}$ was estimated from the detrended residuals on control points and was set equal to the standard deviation squared of the residuals. Empirical and analytical covariances of the detrended residuals for ANN surface and EGG97 quasigeoid of the fourth test (99 control points) are shown in Figure 3. The covariances were plotted to a distance of $150 \mathrm{~km}$ and a step of $10 \mathrm{~km}$ was used as the interval. Covariances are in $[\mathrm{cm}]^{2}$ and distances are in kilometers. The results are presented in Table 3 and correlation distance was estimated at $\xi=17 \mathrm{~km}$.
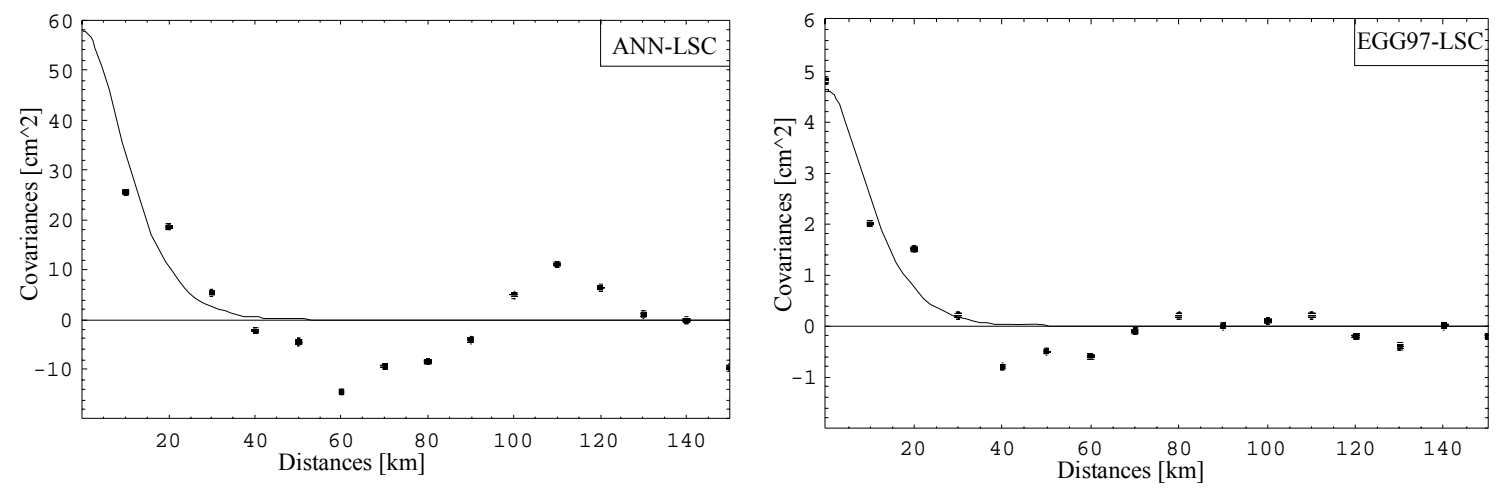

Fig. 3. Empirical covariances for ANN-surface and EGG97

The estimated quasigeoid undulations $\zeta^{E S T}$ without and with the LSC on validation points were compared with the actual quasigeoid undulations $\zeta^{G P S}$. The mean, the standard deviation, the minimum and the maximum of differences between $\zeta^{E S T}$ and $\zeta^{G P S}$, together with the correlation coefficients between them were computed in each test. In Table 2 the ANN approximation is compared to EGM96, EGG97 and MC models, while the effects of LSC using the ANN, EGM96 and EGG97 as trend functions are shown in Table 3.

The comparison shown in Tables 2 and 3 reveals that the EGG97 in each test gives the minimum standard deviation and the highest correlation coefficient. Even without the LSC, the EGG97 gives the quasigeoid heights with cm-level accuracy (see also [2]). If the number of control points is low, the effect of the LSC on the ANN approximation is not critical. If the number of control points is higher, the results of the ANN approximation improve considerably. Not surprisingly, this is because the more training points there are, the better is the learning process and the estimated values for trend in validation points. In these cases the LSC improves the results of the ANN approximation as well. In the case of the MC-solution, the number of control points does not considerably effect the final results. In the fourth test with 99 control points the standard deviation of the MC-solution even decreases. The reason may be in the low number of validation points (26 compared with 50 in the third test).

The number of control points does not have the same effect when either the EGM96 or the EGG97 model is used as a trend surface. In the case of the EGG97 the contribution of the interpolated residuals is negligible (Table 2), confirming the statistical statement about low number of validation points in each consecutive test $(100,75,50,26)$. 
Table 2. Comparison of $\zeta^{G P S}$ and $\zeta^{E S T}$ (without LSC)

\begin{tabular}{crcccc}
\hline \multicolumn{5}{c}{ Number of control points: 25 } \\
\hline Solution & Mean & St. Dev. & Min. & Max. & Correl. \\
\hline ANN & -0.006 & 0.171 & -0.703 & 0.349 & 0.97830 \\
EGM96 & 0.019 & 0.229 & -0.527 & 0.556 & 0.95350 \\
EGG97 & -0.001 & 0.022 & -0.052 & 0.074 & 0.99961 \\
MC & 0.003 & 0.165 & -0.745 & 0.331 & 0.97717 \\
\hline \multicolumn{5}{c}{ Number of control points: 50 } \\
\hline Solution & Mean & St. Dev. & Min. & Max. & Correl. \\
\hline ANN & -0.010 & 0.127 & -0.305 & 0.360 & 0.98701 \\
EGM96 & 0.006 & 0.220 & -0.562 & 0.515 & 0.95893 \\
EGG97 & -0.001 & 0.021 & -0.054 & 0.072 & 0.99963 \\
MC & 0.005 & 0.095 & -0.294 & 0.280 & 0.99252 \\
\hline \multicolumn{5}{c}{ Number of control points: 75 } \\
\hline Solution & Mean & St. Dev. & Min. & Max. & Correl. \\
\hline ANN & -0.001 & 0.079 & -0.197 & 0.148 & 0.99495 \\
EGM96 & 0.012 & 0.235 & -0.572 & 0.487 & 0.95372 \\
EGG97 & -0.002 & 0.021 & -0.043 & 0.059 & 0.99967 \\
MC & -0.003 & 0.069 & -0.204 & 0.128 & 0.99637 \\
\hline \multicolumn{5}{c}{ Number of control points: 99 } \\
\hline Solution & Mean & St. Dev. & Min. & Max. & Correl. \\
\hline ANN & 0.001 & 0.067 & -0.126 \\
EGM96 & 0.036 & 0.218 & -0.344 & 0.113 & 0.99633 \\
EGG97 & 0.007 & 0.023 & -0.021 & 0.079 & 0.96224 \\
MC & -0.011 & 0.083 & -0.140 & 0.99964 \\
\hline \multicolumn{5}{c}{0.298} & 0.99435 \\
\hline
\end{tabular}

Table 3. Comparison of $\zeta^{G P S}$ and $\zeta^{E S T}$ (LSC included)

\begin{tabular}{cccccc}
\hline \multicolumn{5}{c}{ Number of control points: 25 } \\
\hline Solution & Mean & St. Dev. & Min. & Max. & Correl. \\
\hline ANN & -0.011 & 0.179 & -0.742 & 0.389 & 0.97715 \\
EGM96 & -0.010 & 0.148 & -0.518 & 0.464 & 0.98094 \\
EGG97 & -0.002 & 0.018 & -0.066 & 0.057 & 0.99972 \\
\hline \multicolumn{5}{c}{ Number of control points: 50 } \\
\hline Solution & Mean & St. Dev. & Min. & Max. & Correl. \\
\hline ANN & -0.010 & 0.123 & -0.321 \\
EGM96 & -0.003 & 0.080 & -0.312 & 0.394 & 0.98810 \\
EGG97 & -0.001 & 0.015 & -0.048 & 0.060 & 0.99473 \\
\hline \multicolumn{5}{c}{ Number of control points: 75 } \\
\hline Solution & Mean & St. Dev. & Min. & Max. & Correl. \\
\hline ANN & -0.011 & 0.055 & -0.144 \\
EGM96 & -0.010 & 0.067 & -0.171 & 0.135 & 0.99756 \\
EGG97 & -0.002 & 0.013 & -0.028 & 0.110 & 0.99721 \\
\hline \multicolumn{5}{c}{ Number of control points: 99 } \\
\hline Solution & Mean & St. Dev. & Min. & Max. & Correl. \\
\hline ANN & -0.008 & 0.043 & -0.094 \\
EGM96 & -0.011 & 0.067 & -0.149 & 0.061 \\
EGG97 & 0.002 & 0.018 & -0.022 & 0.99849 \\
\hline \multicolumn{7}{c}{0.067} \\
\hline
\end{tabular}


For the two tests with 50 control points and 99 control points contour plots of the differences are presented (Figure 4 and Figure 5).
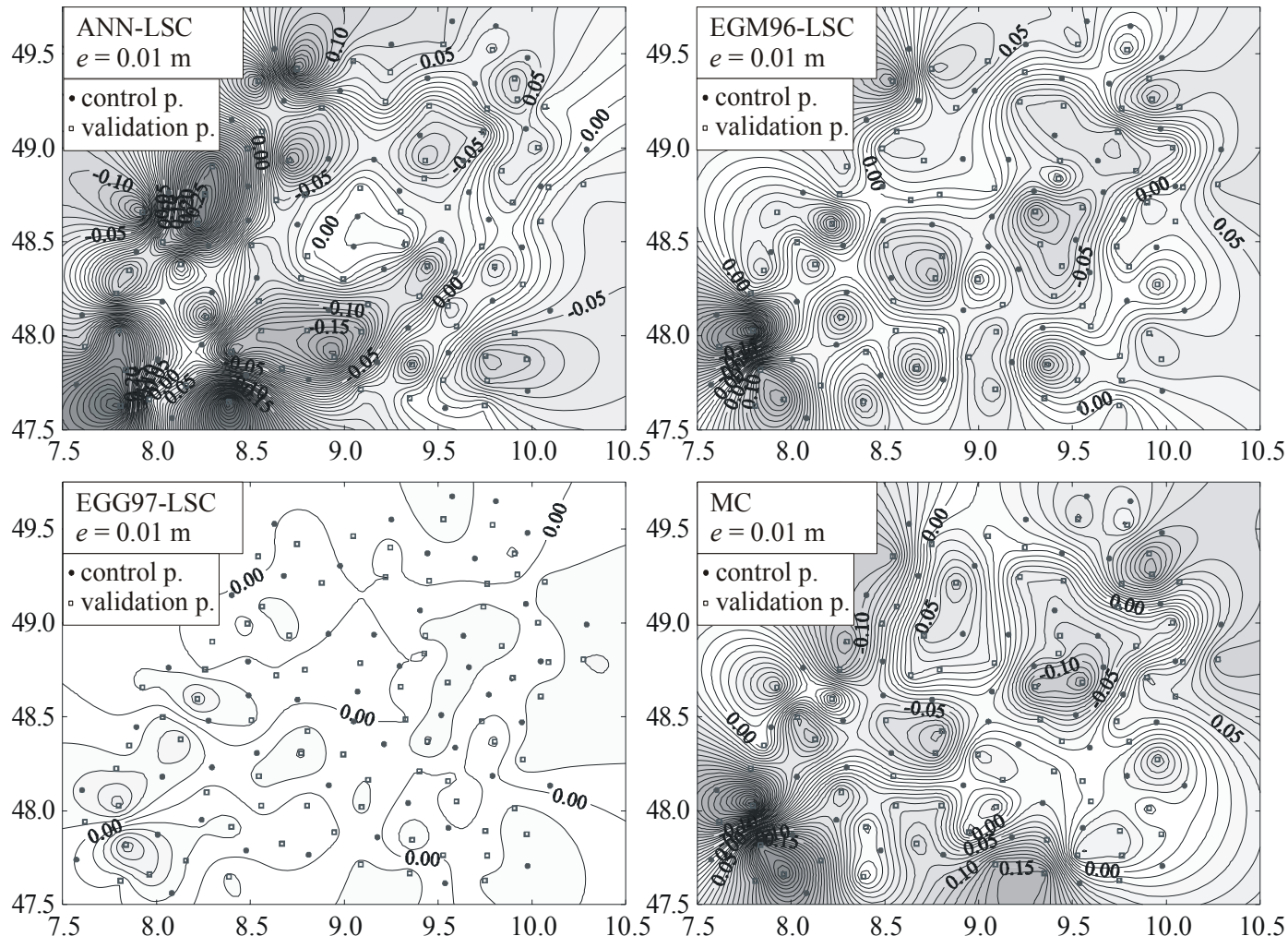

Fig. 4. Differences between control and validation points (50 control points)
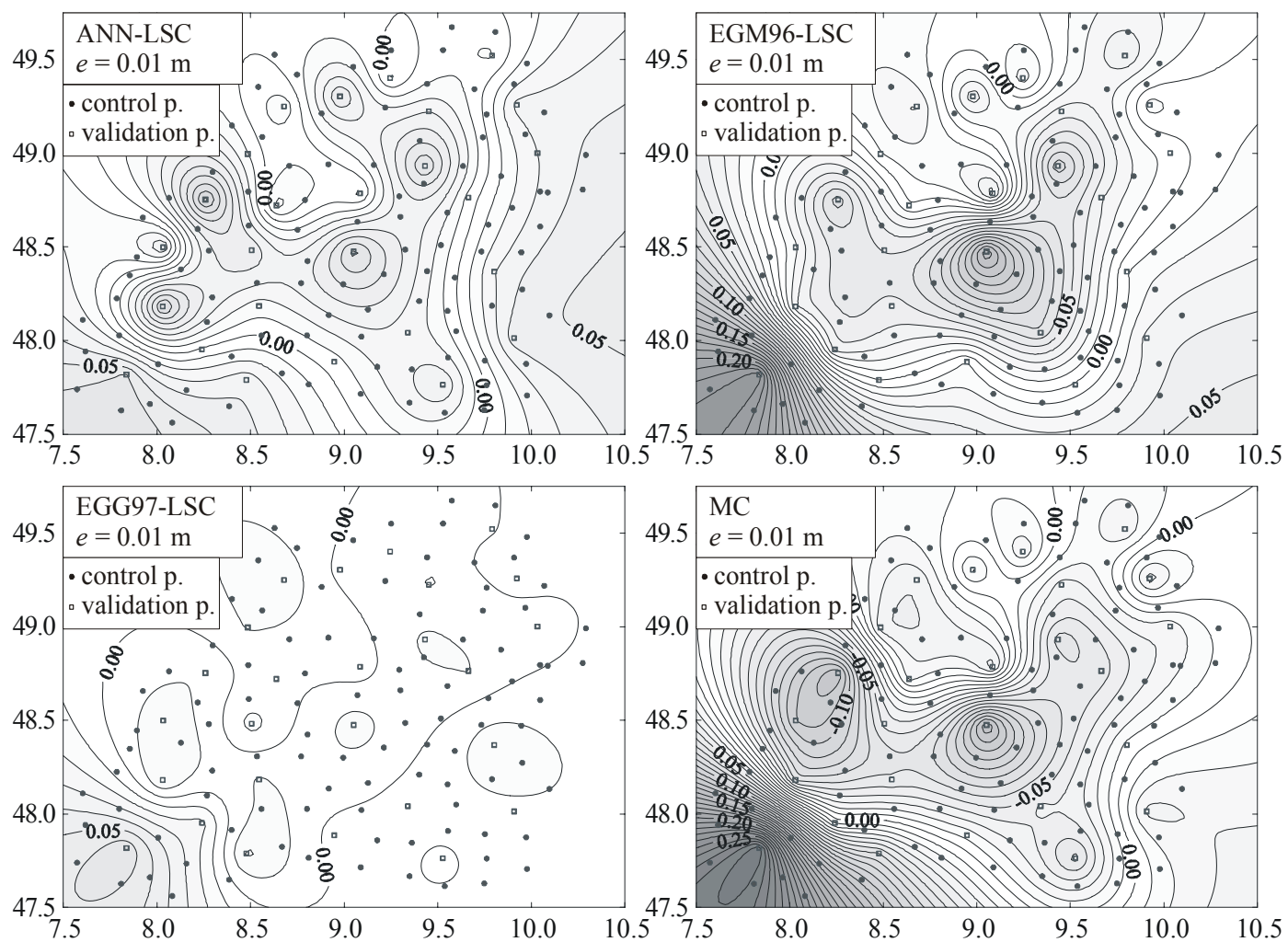

Fig. 5. Differences between control and validation points (99 control points) 


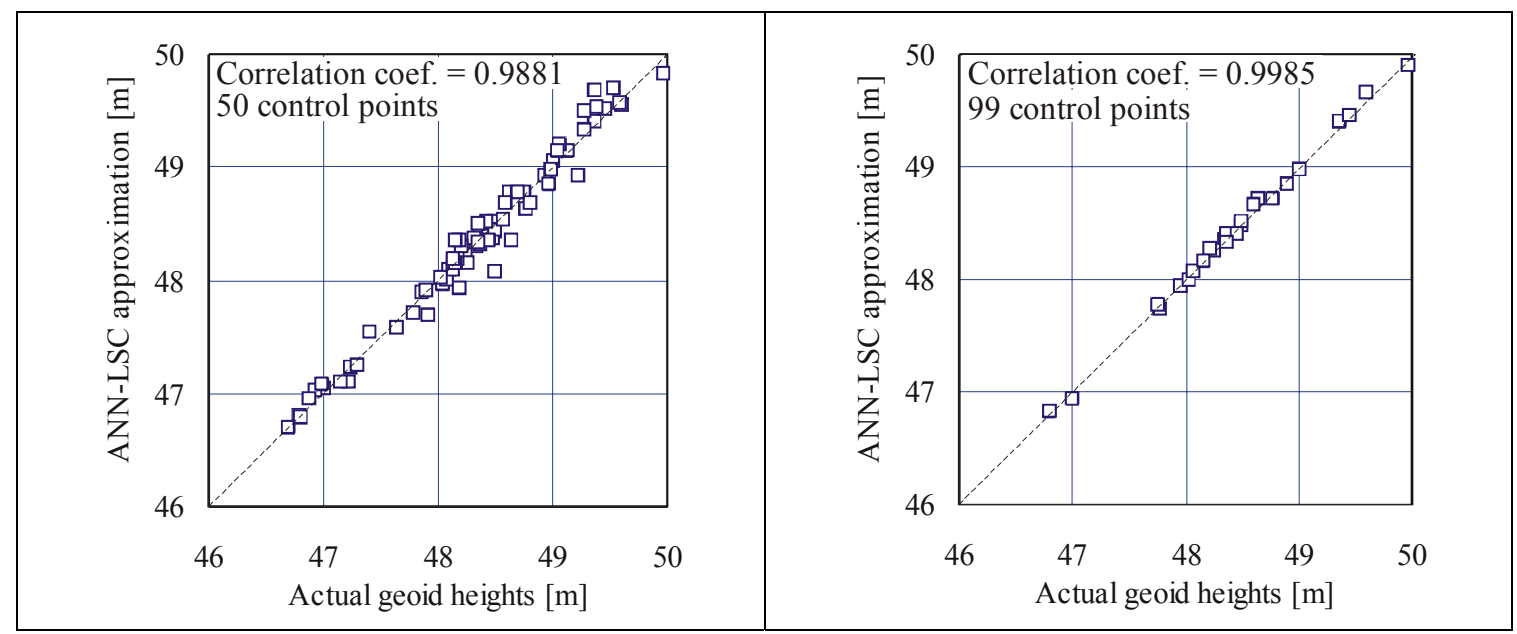

Fig. 6. Correlation coefficents in ANN-LSC approximation

In the case of the EGG97-LSC, there is almost a lack of improvement in spite of an increased number of control points. In the case of the EGM96-LSC the results slightly improve by increasing the number of control points. As seen from Figures 4, 5 and 6 the results obtained with the ANN-LSC model improve considerably when increasing the number of training (control) points.

\section{CONCLUSION}

The ANN approach of determining the GPS-derived quasigeoid heights is completely different from EGM96 and EGG97 geoid models but in some way similar to the minimum curvature splines in the tension algorithm. This is why it is not surprising that the $\mathrm{MC}$ an the ANN give similar results. However, unlike $\mathrm{MC}$, the results with ANN-LSC improve with the increasing number of height control points.

If a fairly large number of control points is available, the ANN-LSC method can effectively be used. This may be considered as an advantage, if the geoid or quasigeoid model in the area of interest has not been validated yet. The ANN-LSC method would give optimal results if the control points were well distributed over the area. From the results obtained it can be concluded that the ANN-LSC approximation gives as accurate results as the MC-surface and fairly better results when a higher number of control points is available.

The global geopotential model EGM96 should be used only if there are no alternatives. Undoubtedly, for the area under consideration the EGG97 model is a highly accurate model, and recommended, since for the test area a large amount of gravimetric data was used for its determination.

Thus, the ANN-LSC method could be a valid alternative to the existing geoid or quasigeoid models, specifically for the areas with no accurate models available. The GPS-derived geoid heights with the ANN-LSC will provide additional reliable information about the geoid shape.

\section{ACKNOWLEDGMENT}

Institut für Erdmessung (Hannover) and IAG are kindly acknowledged for providing the European Gravimetric Quasigeoid 1997 (EGG97). 


\section{References}

1. Ambrožič, T., Kuhar, M., Stopar, B. and Turk, G., 1999. Approximation of Local Geoid Surface by Artificial Neural Network. Proc. of Geodesy and Surveying in the Future - The Importance of Heights. NLS. Gävle. 273-283.

2. Denker, H., 1998. Evaluation and Improvement of the EGG97 Quasigeoid Model for Europe by GPS and Levelling data. Proc. of Second Continental Workshop on the Geoid in Europe. Budapest. 53-61.

3. Denker, H. and Torge, W., 1998. The European Gravimetric Quasigeoid EGG97 - an IAG Supported Continental Enterprise. Proc. of IAG 1997 Scientific Assembly. Rio de Janeiro. Springer. 249-254.

4. Funahashi, K., 1989. On the Approximate Realization of Continuous Mappings by Neural Networks. Neural Networks [2]: 183-192.

5. Goldberg, D.E., 1989. Genetic Algorithms in Search, Optimization, and Machine Learning. Addison-Wesley Publishing Company, Reading. 412 pages.

6. Golden Software Inc, 1998. Surfer for Windows, Version 8 User's Guide. Golden Software Inc. Golden. 640 pages.

7. Hornik, K., Stinchcombe, M. and White, H., 1989. Multilayer Feedforward Networks are Universal Approximators. Neural Networks [2]: 359-366.

8. Hu, W., Kong, Y., Deng, Y. and Huang, S., 2002. A New Method to Derive Normal Height from GPS Height Based on Neural Network. FIG XXII International Congress.Washington DC. TS5.2.

9. James, G.K. and Ojo, A.K., 2001. Artificial Neural Networks for Geoidal Undulation Prediction. Proc. of IAG 2001 Scientific Assembly. Budapest. 25.

10. Janakiraman, J. and Honavar, V., 1993. Adaptive Learning Rate for Increasing Learning Speed in Backpropagation Networks. Proc. of the World Congress on Neural Networks. Portland. IV-378IV-381.

11. Jordan, S.K., 1972. Self-consistent Statistical Models for the Gravity Anomaly, Vertical Deflections and Undulations of the Geoid. Journal of Geophysical Research [77]: 3660-3670.

12. Lemoine, F.G., Kenyon, S.C., Factor, J.K., Trimmer, R.G., Pavlis, N.K., Schin, D.S., Cox, C.M., Klosko, S.M., Luthcke, S.B., Torrence, M.H., Wang, Y.M., Williamson, R.G., Pavlis, E.C., Rapp, R.H. and Olson, T.R., 1998. The Development of the joint NASA GSFC and National Imagery and Mapping Agency (NIMA) Geopotential Model EGM96. NASA/TP-1998-206861, Goddard Space Flight Center, Greenbelt.

13. Lippmann, R.P., 1987. An Introduction to Computing with Neural Nets. IEEE ASSP Mag [4] (2): 4-22.

14. Mikhail, E.M. and Ackerman, F., 1976. Observations and Least Squares. University Press of America, Lanham New York London. 497 pages.

15. Moritz, H.,1980. Advanced Physical Geodesy. Herbert Wichmann Verlag, Karlsruhe. 500 pages.

16. Rumelhart, D.E. and McClelland, J.L., 1986. Parallel Distributed Processing. Volume 1: Foundations. The MTI Press, Cambridge. 547 pages.

17. Sarle, W.S., 2002. Neural Network FAQ. Periodic posting to the Usnet newsgroup comp.ai.neuralnets, URL: ftp://ftp.sas.com/pub/neural/FAQ.html

18. Seager, J.S., Collier, P. and Kirby, J.F., 1999. Modelling Geoid Undulations with an Artificial Neural Network. Proc. of the IEEE International Joint Conference on Neural Networks. Washington DC. 3332-3335.

19. Smith, W.H.F. and Wessel, P., 1990. Gridding with continous curvature splines in tension. Geophysics [55] (10): 293-305.

20. Treadgold, N.K., Gedeon, T.D., 1998. Simulated Annealing and Weight Decay in Adaptive Learning. IEEE Transactions on Neural Networks [9]: 662-668.

21. Wenzel, H.G., 1999. GPS/Levelling Derived Height Anomalies or Geoid Heights. URL: http://www.gik.uni-Karlsruhe.de/wenzel/gpslevha/bwref97b.dat 